Document downloaded from:

http://hdl.handle.net/10251/139766

This paper must be cited as:

Hueso, JL.; Martínez Molada, E.; Teruel-Ferragud, C. (02-0). Multipoint efficient iterative methods and the dynamics of Ostrowski's method. International Journal of Computer Mathematics. 96(9):1687-1701. https://doi.org/10.1080/00207160.2015.1080354

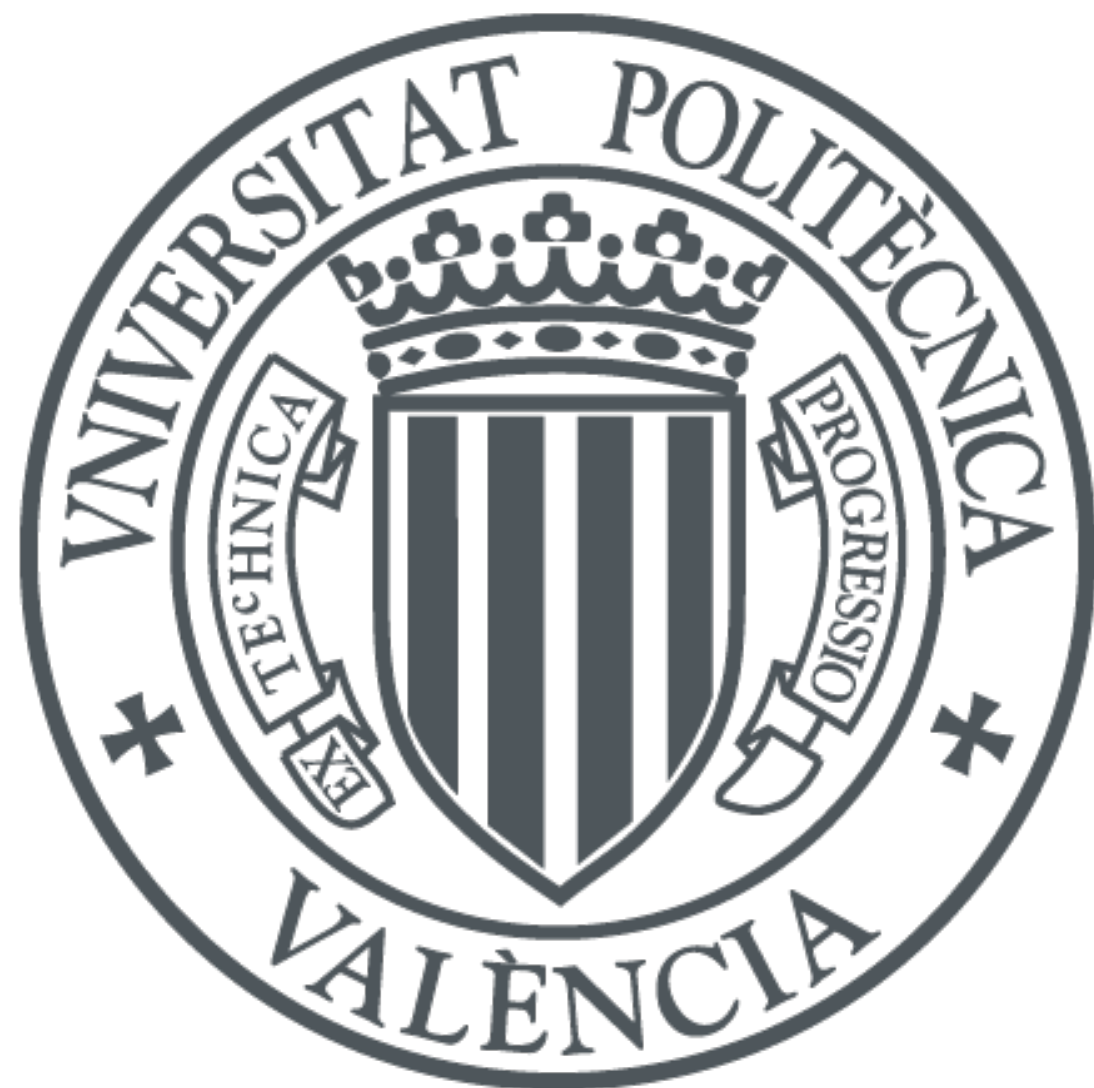

The final publication is available at

https://doi.org/10.1080/00207160.2015.1080354

Copyright Taylor \& Francis

Additional Information

This is an Author's Accepted Manuscript of an article published in José L. Hueso, Eulalia Martínez \& Carles Teruel (2019) Multipoint efficient iterative methods and the dynamics of Ostrowski's method, International Journal of Computer Mathematics, 96:9, 1687-1701, DOI: 10.1080/00207160.2015.1080354 in the International Journal of Computer Mathematics, SEP 22019 [copyright Taylor \& Francis], available online at:

http://www.tandfonline.com/10.1080/00207160.2015.1080354 


\title{
Multipoint efficient iterative methods and the dynamics of Ostrowski's method*
}

\author{
José L. Hueso $^{(a)}$, Eulalia Martínez ${ }^{(a) \dagger}$ Carles Teruel $^{(b)}$ \\ ${ }^{(a)}$ Instituto Universitario de Matemática Multidisciplinar \\ ${ }^{(a, b)}$ Universitat Politècnica de València \\ Spain
}

May 2, 2019

\begin{abstract}
In this work we introduce a modification into the technique, presented in [1], that increases by two units the convergence order of an iterative method.

The main idea is to compose a given iterative method of order $p$ with a modification of Newton's method that introduces just one evaluation of the function, obtaining a new method of order $p+2$, avoiding the need to compute more than one derivative, so we improve the efficiency index in the scalar case.

This procedure can be repeated $n$ times, with the same approximation to the derivative, obtaining new iterative methods of order $p+2 n$.

We perform different numerical tests that confirm the theoretical results.

By applying this procedure to Newton's method one obtains the well known fourth order Ostrowski's method. We finally analyze its dynamical behaviour on second and third degree real polynomials.
\end{abstract}

Keywords: Nonlinear equations; order of convergence; iterative methods; Dynamics.

2010 Mathematics Subject Classification. 65H05, 65D05, 41A25.

\section{Introduction}

One of the most important problems in numerical analysis is approximating the solution of nonlinear equations by using iterative methods. Let us consider the problem of finding a zero of a function $f: \mathbb{R} \longrightarrow \mathbb{R}$, that is, a solution $\alpha$ of the

*This research was supported by Ministerio de Economía y Competitividad under grant MTM2014-52016-C2-1-2-P and by the project of Generalitat Valenciana Prometeo/2016/089.

†Corresponding author, eumarti@mat.upv.es 
nonlinear equation $f(x)=0$. The best known iterative method is the classical Newton's method, which converges quadratically under certain conditions. Many higher order robust and efficient methods have been proposed [2, 3], as well as techniques that allow us to increase the convergence order, see among others $[4,5]$.

As the order of an iterative method increases, so does the number of functional evaluations per step. The efficiency index (see [3]), gives a measure of the balance between those quantities, according to the formula $p^{1 / n}$, where $p$ is the convergence order of the method and $n$ the number of function evaluations per step. Kung and Traub [6] conjecture that, in this case, the order of convergence of any multipoint method without memory cannot exceed the bound $2^{n-1}$, then, if a method that performs $n$ functional evaluations reaches this order it is call optimal method, opposite the method is non-optimal.

Traub [2] introduces a technique for increasing the convergence order of an iterative method by one unit by using the derivative computed in the first step. In [1], we improved this idea presenting a procedure that increases the convergence order by two units.

Our aim in this paper is to introduce an improvement of this technique which efficiently increases the convergence order of an iterative method in the unidimensional case. The main idea is to compose a given iterative method of order $p$ with a modification of Newton's method that introduces just one evaluation of the function, obtaining a new method of order $p+2$, avoiding the need to compute more than one derivative. Thus by getting an approximation for the derivative in the second step using previously evaluated functions.

We also study the dynamics of some Newton-type iterative methods obtained with this technique, when they are applied to real polynomials of degrees two and three and compare them with the study made in [7].

The rest of the paper is organized as follows. In section 2, we prove the convergence order result for the improvement technique. Then, we apply this technique to standard iterative methods, obtaining new ones, some of which are optimal. The same idea can be used with methods whose first step is not a Newton's step, as it is explained for Jarratt's method. In section 3, several particular methods are applied to some test equations. Finally, section 4 examines the dynamics of one of these methods and section 5 is devoted to the conclusions.

\section{Main Result}

Several authors have developed techniques to increase the order of an iterative method by performing new Newton-like steps. For example, it is known that freezing the derivative of the first step of Newton's method, a multistep Newton's method is obtained. The following result generalizes this idea:

Theorem 1 (Traub [2]) Assume that the function $f: D \subset \mathbb{R} \longrightarrow \mathbb{R}$ defined on an open interval $D$ has a simple root $x^{*} \in D$ and is sufficiently smooth. If the 
iterative function $\phi(x)$ defines an iterative method of order $p$, then the composite iterative function $\psi(x)$ given by

$$
\psi(x)=\phi(x)-\frac{f(\phi(x))}{f^{\prime}(x)}
$$

defines an iterative method of order $p+1$.

Furthermore, in the case of nonlinear systems, Cordero, et al.[1] have proved a similar statement by reusing the derivative in the second step:

Theorem 2 Let $F: \mathbb{R}^{n} \longrightarrow \mathbb{R}^{n}$ be a sufficiently differentiable function in a neighborhood $D$ of $\alpha$, that is a solution of the system $F(x)=0$, whose Jacobian matrix is continuous and nonsingular in $D$. Then, for an initial approximation sufficiently close to $\alpha$, the method defined by

$$
\psi(x)=\phi(x, y)-F^{\prime}(y)^{-1} F(\phi(x, y))
$$

has order of convergence $p+2$, where $y=x-F^{\prime}(x)^{-1} F(x)$ is the iteration of Newton's method and $\phi(x, y)$ is the iteration function of a method of order $p$.

Here we are interested in improving this technique, for the scalar case, by avoiding the computation of the derivative at $y$. Thus, we will use an approximation of the derivative that allows us to maintain the increase in the order of convergence. The technique presented in this paper can be expressed in a two-step iterative method as follows:

$$
\begin{aligned}
y_{k} & =x_{k}-\frac{f\left(x_{k}\right)}{f^{\prime}\left(x_{k}\right)} \\
z_{k} & =\phi\left(x_{k}, y_{k}\right) \\
x_{k+1} & =z_{k}-\frac{f\left(z_{k}\right)}{\widetilde{f}^{\prime}\left(y_{k}\right)}
\end{aligned}
$$

where $\tilde{f}^{\prime}\left(y_{k}\right)$ is an approximation to the derivative.

This approximation can be deduced from the Taylor's expansion of $f\left(y_{k}\right)$ truncated in the second degree:

$$
f\left(y_{k}\right) \simeq f\left(x_{k}\right)+f^{\prime}\left(x_{k}\right)\left(y_{k}-x_{k}\right)+\frac{f^{\prime \prime}\left(x_{k}\right)}{2 !}\left(y_{k}-x_{k}\right)^{2}
$$

From here and using that, since $y_{k}$ is a Newton's step, $y_{k}-x_{k}=-\frac{f\left(x_{k}\right)}{f^{\prime}\left(x_{k}\right)}$, we have:

$$
f^{\prime \prime}\left(x_{k}\right) \simeq \frac{2 f\left(y_{k}\right) f^{\prime}\left(x_{k}\right)^{2}}{f\left(x_{k}\right)^{2}}
$$


Using this expression in the linear Taylor's expansion of $f^{\prime}\left(y_{k}\right)$ we obtain:

$$
\begin{aligned}
f^{\prime}\left(y_{k}\right) & \simeq f^{\prime}\left(x_{k}\right)+f^{\prime \prime}\left(x_{k}\right)\left(y_{k}-x_{k}\right) \\
& =\left(f\left(x_{k}\right)-2 f\left(y_{k}\right)\right) \frac{f^{\prime}\left(x_{k}\right)}{f\left(x_{k}\right)}=\tilde{f}^{\prime}\left(y_{k}\right)
\end{aligned}
$$

This approximation leads us to the following result:

Theorem 3 Let $f: \mathbb{R} \longrightarrow \mathbb{R}$ be sufficiently smooth function in a neighborhood $D$ of $\alpha$, where $\alpha$ is a single root of the equation $f(x)=0$. If $\phi$ is a method of order $p$, then the method (2), where $\tilde{f}^{\prime}\left(y_{k}\right)$ is given by (3), has convergence order $p+2$, assuming that the initial guess is sufficiently close to $\alpha$.

Proof: Consider the Taylor's expansion of $f\left(x_{k}\right)$ around the solution $\alpha$

$$
f\left(x_{k}\right)=c_{1} e_{k}+c_{2} e_{k}^{2}+c_{3} e_{k}^{3}+c_{4} e_{k}^{4}+O\left(e_{k}^{5}\right)
$$

where $e_{k}=x_{k}-\alpha$ and $c_{n}=\frac{f^{(n)}(\alpha)}{n !}, n \geq 1$ and its derivative

$$
f^{\prime}\left(x_{k}\right)=c_{1}+2 c_{2} e_{k}+3 c_{3} e_{k}^{2}+4 c_{4} e_{k}^{3}+5 c_{5} e_{k}^{4}+O\left(e_{k}^{5}\right)
$$

Then, the Newton's step can be written as

$y_{k}-\alpha=e_{k}-\frac{f\left(x_{k}\right)}{f^{\prime}\left(x_{k}\right)}=\frac{c_{2}}{c_{1}} e_{k}^{2}+\frac{2\left(c_{1} c_{3}-c_{2}^{2}\right)}{c_{1}^{2}} e_{k}^{3}+\frac{\left(4 c_{2}^{3}-7 c_{1} c_{3} c_{2}+3 c_{1}^{2} c_{4}\right)}{c_{1}^{3}} e_{k}^{4}+O\left(e_{k}^{5}\right)$

and performing Taylor's development of $f\left(y_{k}\right)$ around $\alpha$ by using the value $y_{k}-\alpha$ already obtained we have:

$$
f\left(y_{k}\right)=c_{2} e_{k}^{2}+\left(2 c_{3}-\frac{2 c_{2}^{2}}{c_{1}}\right) e_{k}^{3}+\left(\frac{5 c_{2}^{3}}{c_{1}^{2}}-\frac{7 c_{3} c_{2}}{c_{1}}+3 c_{4}\right) e_{k}^{4}+O\left(e_{k}^{5}\right) .
$$

Substituting these expressions in (3), the approximation built for $f^{\prime}\left(y_{k}\right)$ takes the form:

$$
\begin{aligned}
\tilde{f}^{\prime}\left(y_{k}\right) & =\left(f\left(x_{k}\right)-2 f\left(y_{k}\right)\right) \frac{f^{\prime}\left(x_{k}\right)}{f\left(x_{k}\right)} \\
& =c_{1}+\left(\frac{2 c_{2}^{2}}{c_{1}}-c_{3}\right) e_{k}^{2}+\left(-\frac{4 c_{2}^{3}}{c_{1}^{2}}+\frac{6 c_{3} c_{2}}{c_{1}}-2 c_{4}\right) e_{k}^{3} \\
& +\left(\frac{8 c_{2}^{4}}{c_{1}^{3}}-\frac{16 c_{3} c_{2}^{2}}{c_{1}^{2}}+\frac{4\left(c_{3}^{2}+2 c_{2} c_{4}\right)}{c_{1}}-3 c_{5}\right) e_{k}^{4}+O\left(e_{k}^{5}\right)
\end{aligned}
$$

If $z_{k}=\phi\left(x_{k}, y_{k}\right)$ is a method of order $p$, its error expression has the form:

$$
z_{k}-\alpha=B_{p} e_{k}^{p}+B_{p+1} e_{k}^{p+1}+B_{p+2} e_{k}^{p+2}+O\left(e_{k}^{p+3}\right)
$$


and then

$f\left(z_{k}\right)=B_{p} c_{1} e_{k}^{p}+B_{p+1} c_{1} e_{k}^{p+1}+B_{p+2} c_{1} e_{k}^{p+2}+O\left(e_{k}^{p+3}\right)+c_{2} B_{p}^{2} e_{k}^{2 p}+O\left(e_{k}^{2 p+1}\right)$,

where $B_{p}, B_{p+1}, B_{p+2} \ldots$ are real coefficients that depend on the specific method implemented.

We have two cases. If $p=2$,

$$
f\left(z_{k}\right)=B_{2} c_{1} e_{k}^{2}+B_{3} c_{1} e_{k}^{3}+\left(B_{2}^{2} c_{2}+B_{4} c_{1}\right) e_{k}^{4}+O\left(e_{k}^{5}\right),
$$

then

$$
\frac{f\left(z_{k}\right)}{\widetilde{f}^{\prime}\left(y_{k}\right)}=B_{2} e_{k}^{2}+B_{3} e_{k}^{3}+\frac{B_{4} c_{1}+B_{2}\left(B_{2} c_{2}-\frac{2 c_{2}^{2}}{c_{1}}+c_{3}\right)}{c_{1}} e_{k}^{4}+O\left(e_{k}^{5}\right)
$$

and the error expression takes the form:

$$
e_{k+1}=\left(z_{k}-\alpha\right)-\frac{f\left(z_{k}\right)}{\widetilde{f}^{\prime}\left(y_{k}\right)}=\frac{B_{2}}{c_{1}}\left(B_{2} c_{2}+\left(\frac{2 c_{2}^{2}}{c_{1}}-c_{3}\right)\right) e_{k}^{4}+O\left(e_{k}^{5}\right) .
$$

If $p>2$,

$$
f\left(z_{k}\right)=B_{p} c_{1} e_{k}^{p}+B_{p+1} c_{1} e_{k}^{p+1}+B_{p+2} c_{1} e_{k}^{p+2}+O\left(e_{k}^{p+3}\right),
$$

then

$$
\frac{f\left(z_{k}\right)}{\widetilde{f}^{\prime}\left(y_{k}\right)}=B_{p} e_{k}^{p}+B_{p+1} e_{k}^{p+1}+\frac{1}{c_{1}}\left(B_{p+2} c_{1}-B_{p}\left(\frac{2 c_{2}^{2}}{c_{1}}-c_{3}\right)\right) e_{k}^{p+2}+O\left(e_{k}^{p+3}\right)
$$

and the error expression is:

$$
e_{k+1}=\left(z_{k}-\alpha\right)-\frac{f\left(z_{k}\right)}{\widetilde{f}^{\prime}\left(y_{k}\right)}=\frac{B_{p}\left(2 c_{2}^{2}-c_{1} c_{3}\right)}{c_{1}^{2}} e_{k}^{p+2}+O\left(e_{k}^{p+3}\right) .
$$

In both cases, the convergence order is at least $p+2$.

\subsection{New iterative methods}

This result us allows to obtain new methods that increase the order of convergence of given iterative methods of convergence order $p$. By applying the procedure $n$ times, one has a $p+2 n$-th order method of the form:

$$
\begin{aligned}
y_{k 0} & =x_{k} \\
y_{k 1} & =y_{k 0}-\frac{f\left(y_{k 0}\right)}{f^{\prime}\left(y_{k 0}\right)} \\
y_{k 2} & =\phi\left(y_{k 0}, y_{k 1}\right) \\
y_{k, j+1} & =y_{k j}-\frac{f\left(y_{k j}\right)}{\widetilde{f}^{\prime}\left(y_{k 1}\right)} ; \quad j=2,3, \ldots, n+1 \\
x_{k+1} & =y_{k, j+1}
\end{aligned}
$$


Specifically, we apply the presented technique to the second order Newton's method, the third order method given in [2] and the fourth order method from [4]. The new methods will be referred to with a letter and the numbers of steps added with our procedure.

1.- Taking $\phi\left(y_{k 0}, y_{k 1}\right)=y_{k 1}$ results in repeatedly applying the procedure to Newton's method, obtaining methods $N n$ of order of convergence $2+2 n$.

2.- From the third order Traub's method [2], $(3+2 n)$-th order methods Tn can be obtained, by taking

$$
\phi\left(y_{k 0}, y_{k 1}\right)=y_{k 0}-\frac{f\left(y_{k 0}\right)+f\left(y_{k 1}\right)}{f^{\prime}\left(y_{k 0}\right)} .
$$

3.- From the fourth order method [4], we get order $4+2 n$ method for

$$
\phi\left(y_{k 0}, y_{k 1}\right)=y_{k 1}-\left(2-\frac{f^{\prime}\left(y_{k 1}\right)}{f^{\prime}\left(y_{k 0}\right)}\right) \frac{f\left(y_{k 1}\right)}{f^{\prime}\left(y_{k 0}\right)} \text {. }
$$

Observe that Newton's method with a step of our procedure, $N 1$, is the optimal Ostrowski's method, [3], and that N2 is the algorithm proposed by Grau and Diaz-Barrero in [9], obtained here in a different way.

\subsection{New optimal methods}

This technique can be applied to some methods which use $f\left(y_{k}\right)$ and $f^{\prime}\left(y_{k}\right)$ avoiding the evaluation of the derivative and obtaining optimal methods. For example, the 4-th order method defined in [4] can be optimized as follows:

$$
\begin{aligned}
y_{k} & =x_{k}-\frac{f\left(x_{k}\right)}{f^{\prime}\left(x_{k}\right)} \\
x_{k+1} & =y_{k}-\left(2-\frac{\tilde{f}^{\prime}\left(y_{k}\right)}{f^{\prime}\left(x_{k}\right)}\right) \frac{f\left(y_{k}\right)}{f^{\prime}\left(x_{k}\right)}
\end{aligned}
$$

because we only perform 3 functional evaluation per step. In the same way as before, the order of the method can be increased repeatedly by two units, obtaining methods of order of convergence $4+2 n$.

Theorem 4 Let $f: \mathbb{R} \longrightarrow \mathbb{R}$ be sufficiently smooth function in a neighborhood $D$ of $\alpha$, where $\alpha$ is a single root of the equation $f(x)=0$. Then the method (8), where $\tilde{f}^{\prime}\left(y_{k}\right)$ is given by (3), has convergence order 4 , assuming that the initial guess is sufficiently close to $\alpha$, and the method is optimal.

Proof: Proceeding as before, we expand $f\left(x_{k}\right), f^{\prime}\left(x_{k}\right), f\left(y_{k}\right), \tilde{f}^{\prime}\left(y_{k}\right)$ in terms of $e_{k}$ and obtain the error expression:

$$
\begin{aligned}
e_{k+1} & =\left(y_{k}-\alpha\right)-\left(2-\frac{f^{\prime}\left(y_{k}\right)}{f^{\prime}\left(x_{k}\right)}\right) \frac{f\left(y_{k}\right)}{f^{\prime}\left(x_{k}\right)}= \\
& =\frac{\left(5 c_{2}^{3}-c_{1} c_{2} c_{3}\right)}{c_{1}^{3}} e_{k}^{4}-\frac{2\left(18 c_{2}^{4}-16 c_{1} c_{3} c_{2}^{2}+c_{1}^{2} c_{4} c_{2}+c_{1}^{2} c_{3}^{2}\right)}{c_{1}^{4}} e_{k}^{5}+O\left(e_{k}^{6}\right) .
\end{aligned}
$$


In some cases, this technique gets an optimal method from a non-optimal higher order method. This happens, for example, with the fifth order method proposed in [10], which can be optimized as follows:

$$
\begin{aligned}
y_{k} & =x_{k}-\frac{f\left(x_{k}\right)}{f^{\prime}\left(x_{k}\right)} \\
x_{k+1} & =y_{k}-\left[1+\left(\frac{f\left(y_{k}\right)}{f\left(x_{k}\right)}\right)^{2}\right] \frac{f\left(y_{k}\right)}{\widetilde{f^{\prime}}\left(y_{k}\right)} .
\end{aligned}
$$

The resulting method has convergence order 4 with only 3 functional evaluations per step, so that it is optimal. Its efficiency index, $4^{\frac{1}{3}}=1.5874$, is higher than that of the original 5 -th order method, $5^{\frac{1}{4}}=1.4953$. Similarly, the methods of order $4+2 n$ obtained by applying our procedure in the same way as before to the modified method are more efficient than the corresponding methods of order $5+2 n$ derived from the original method.

Theorem 5 Let $f: \mathbb{R} \longrightarrow \mathbb{R}$ be sufficiently smooth function in a neighborhood $D$ of $\alpha$, where $\alpha$ is a single root of the equation $f(x)=0$. Then the method (9), where $\tilde{f}^{\prime}\left(y_{k}\right)$ is given by (3), has convergence order 4 , assuming that the initial guess is sufficiently close to $\alpha$, and the method is optimal.

Proof: Reasoning as before, one gets:

$$
\begin{aligned}
e_{k+1} & =\left(y_{k}-\alpha\right)-\left(1+\left(\frac{f\left(y_{k}\right)}{f\left(x_{k}\right)}\right)^{2}\right) \frac{f\left(y_{k}\right)}{\widetilde{f}^{\prime}\left(y_{k}\right)}= \\
& =-\frac{c_{2} c_{3}}{c_{1}^{2}} e_{n}^{4}+\frac{4 c_{2}^{4}+2 c_{1} c_{3} c_{2}^{2}-2 c_{1}^{2} c_{4} c_{2}-2 c_{1}^{2} c_{3}^{2}}{c_{1}^{4}} e_{n}^{5}+O\left(e_{k}^{6}\right) .
\end{aligned}
$$

\subsection{Jarratt's method}

The previous technique is restricted to methods where the Newton's step is used as a predictor, but a similar procedure can be applied to Jarratt's method, [8]:

$$
\begin{aligned}
& y_{k}=x_{k}-\frac{2}{3} \frac{f\left(x_{k}\right)}{f^{\prime}\left(x_{k}\right)} \\
& z_{k}=x_{k}-\frac{1}{2} \frac{f\left(x_{k}\right)}{f^{\prime}\left(x_{k}\right)} \frac{3 f^{\prime}\left(y_{k}\right)+f^{\prime}\left(x_{k}\right)}{3 f^{\prime}\left(y_{k}\right)-f^{\prime}\left(x_{k}\right)} .
\end{aligned}
$$

From Taylor's expansion:

$$
f\left(z_{k}\right) \simeq f\left(x_{k}\right)+f^{\prime}\left(x_{k}\right)\left(z_{k}-x_{k}\right)+\frac{f^{\prime \prime}\left(x_{k}\right)}{2}\left(z_{k}-x_{k}\right)^{2}
$$


We obtain the expression for the second derivative:

$$
f^{\prime \prime}\left(x_{k}\right) \simeq 2 \frac{f\left(z_{k}\right)-f\left(x_{k}\right)-f^{\prime}\left(x_{k}\right)\left(z_{k}-x_{k}\right)}{\left(z_{k}-x_{k}\right)^{2}}
$$

In Taylor's expansion of $f^{\prime}\left(z_{k}\right)$ :

$$
f^{\prime}\left(z_{k}\right) \simeq f^{\prime}\left(x_{k}\right)+f^{\prime \prime}\left(x_{k}\right)\left(z_{k}-x_{k}\right)
$$

Substituting the expression for $f^{\prime \prime}\left(x_{k}\right)$ and $z_{k}-x_{k}$ :

$f^{\prime}\left(z_{k}\right) \simeq \frac{f^{\prime}\left(x_{k}\right)}{f\left(x_{k}\right)} \frac{f^{\prime}\left(x_{k}\right)\left(4 f\left(z_{k}\right)-5 f\left(x_{k}\right)\right)+3 f^{\prime}\left(y_{k}\right)\left(3 f\left(x_{k}\right)-4 f\left(z_{k}\right)\right)}{3 f^{\prime}\left(y_{k}\right)+f^{\prime}\left(x_{k}\right)}:=\tilde{f}^{\prime}\left(z_{k}\right)$

This approximation allows us to extend the technique to Jarratt's method. So we can obtain from $p$-th order methods, new ones $p+2$-th methods step adding only one functional evaluation.

The error equation is now:

$$
e_{k+1}=\left(z_{k}-\alpha\right)-\frac{f\left(z_{k}\right)}{\widetilde{f}^{\prime}\left(z_{k}\right)}=-\frac{\left(c_{3}\left(9 c_{2}^{3}-9 c_{1} c_{3} c_{2}+c_{1}^{2} c_{4}\right)\right)}{9 c_{1}^{4}} e_{n}^{6}+O\left(e_{k}^{7}\right)
$$

\section{$3 \quad$ Numerical examples}

In this section, we study the performance of the above defined methods for solving the following equations:

$$
\begin{aligned}
f_{1}(x) & =x^{2}+\sin \left(\frac{x}{5}\right)-\frac{1}{4} ; & \alpha & =0.409992017989137 \ldots \\
f_{2}(x) & =10 x e^{-x^{2}}-1 ; & \alpha & =1.679630610428449 \ldots \\
f_{3}(x) & =e^{-x^{2}+x+2}-\cos (x+1)+x^{3}+1 ; & & \alpha=-1 \\
f_{4}(x) & =x e^{x^{2}}-\sin (x)^{2}+3 \cos (x)+5 ; & \alpha & =-1.207647827130919 \ldots \\
f_{5}(x) & =x^{5}+x^{4}+4 x^{2}-15 ; & \alpha & =1.347428098968305 \ldots \\
f_{6}(x) & =\arcsin \left(x^{2}-1\right)-0.5 x+1 ; & \alpha & =0.594810968398369 \ldots
\end{aligned}
$$

The calculations have been performed in MATLAB 2016a by using variable precision arithmetic with 1000 digits and we have used as stopping criterion $\left|x_{k+1}-x_{k}\right|+\left|f\left(x_{k+1}\right)\right| \leq 10^{-100}$. The approximated computational order of convergence (ACOC) defined in [11], is given by :

$$
A C O C=\frac{\ln \left(\left|x_{k+1}-x_{k}\right| /\left|x_{k}-x_{k-1}\right|\right)}{\ln \left(\left|x_{k}-x_{k-1}\right| /\left|x_{k-1}-x_{k-2}\right|\right)} .
$$

The error, the increment of the function and the number of iterations of the above mentioned methods applied to the test equations, are shown in table 1 and 2 . 


\begin{tabular}{|l|l||l|l|l|}
\hline & & $f_{1}$ & $f_{2}$ & $f_{3}$ \\
& & $x_{0}=0.75$ & $x_{0}=1.25$ & $x_{0}=-0.6$ \\
\hline \hline$N 0$ & $\left|x_{k+1}-x_{k}\right|$ & $5.8276 \mathrm{e}-155$ & $9.5288 \mathrm{e}-158$ & $3.5103 \mathrm{e}-130$ \\
& $\left|f\left(x_{k+1}\right)\right|$ & $3.3905 \mathrm{e}-309$ & $2.3992 \mathrm{e}-314$ & $1.2322 \mathrm{e}-259$ \\
& ACOC & 2.0000 & 2.0000 & 2.0000 \\
& iter & 9 & 9 & 8 \\
\hline$N 1$ & $\left|x_{k+1}-x_{k}\right|$ & $6.5389 \mathrm{e}-155$ & $1.8191 \mathrm{e}-134$ & $1.3038 \mathrm{e}-223$ \\
& $\left|f\left(x_{k+1}\right)\right|$ & $1.7533 \mathrm{e}-617$ & $2.4404 \mathrm{e}-535$ & $1.1238 \mathrm{e}-892$ \\
& ACOC & 3.9999 & 3.9999 & 4.0000 \\
& iter & 5 & 5 & 5 \\
\hline$N 2$ & $\left|x_{k+1}-x_{k}\right|$ & $3.0839 \mathrm{e}-119$ & $9.7041 \mathrm{e}-101$ & $4.8346 \mathrm{e}-202$ \\
& $\left|f\left(x_{k+1}\right)\right|$ & $1.5839 \mathrm{e}-711$ & $3.2708 \mathrm{e}-600$ & $1.7932 \mathrm{e}-1209$ \\
& ACOC & 5.9999 & 5.9998 & 6.0000 \\
& iter & 4 & 4 & 4 \\
\hline \hline$T 0$ & $\left|x_{k+1}-x_{k}\right|$ & $3.2188 \mathrm{e}-121$ & $6.7986 \mathrm{e}-125$ & $1.0030 \mathrm{e}-209$ \\
& $\left|f\left(x_{k+1}\right)\right|$ & $6.522 \mathrm{e}-362$ & $1.5876 \mathrm{e}-372$ & $3.3639 \mathrm{e}-628$ \\
& ACOC & 2.9999 & 2.9999 & 3.0000 \\
& iter & 6 & 6 & 6 \\
\hline$T 1$ & $\left|x_{k+1}-x_{k}\right|$ & $7.0611 \mathrm{e}-312$ & $1.4760 \mathrm{e}-288$ & $6.1587 \mathrm{e}-112$ \\
& $\left|f\left(x_{k+1}\right)\right|$ & $6.5909 \mathrm{e}-1556$ & $6.221 \mathrm{e}-1439$ & $1.0665 \mathrm{e}-557$ \\
& ACOC & 4.9999 & 4.9999 & 4.9999 \\
& iter & 5 & 5 & 4 \\
\hline$T 2$ & $\left|x_{k+1}-x_{k}\right|$ & $1.1673 \mathrm{e}-168$ & $6.4574 \mathrm{e}-150$ & $2.0257 \mathrm{e}-322$ \\
& $\left|f\left(x_{k+1}\right)\right|$ & $2.1291 \mathrm{e}-1175$ & $7.3055 \mathrm{e}-1044$ & $6.3262 \mathrm{e}-2254$ \\
& ACOC & 6.9999 & 6.9999 & 7.0001 \\
& iter & 4 & 4 & 4 \\
\hline \hline
\end{tabular}

Table 1: Numerical results from $f_{1}$ to $f_{3}$ for $N n$ and $T n$

\section{The dynamics of Ostrowski's method}

In [7] and [12] the authors analyze the dynamics of a third order method consisting on a two step Newton by using in second step the derivative already evaluated in first step. Here we study the dynamics of method $N 1$, which coincides with the well known optimal iterative Ostrowski's method.

$$
\begin{aligned}
y_{k} & =x_{k}-\frac{f\left(x_{k}\right)}{f^{\prime}\left(x_{k}\right)} \\
x_{k+1} & =y_{k}-\frac{f\left(x_{k}\right) f\left(y_{k}\right)}{\left(f\left(x_{k}\right)-2 f\left(y_{k}\right)\right) f^{\prime}\left(x_{k}\right)}
\end{aligned}
$$




\begin{tabular}{|l|l||l|l|l|}
\hline & & $f_{4}$ & $f_{5}$ & $f_{6}$ \\
& & $x_{0}=-1.3$ & $x_{0}=1.6$ & $x_{0}=1$ \\
\hline \hline$N 0$ & $\left|x_{k+1}-x_{k}\right|$ & $9.1524 \mathrm{e}-112$ & $1.0826 \mathrm{e}-160$ & $7.4779 \mathrm{e}-109$ \\
& $\left|f\left(x_{k+1}\right)\right|$ & $-2.5552 \mathrm{e}-221$ & $4.6127 \mathrm{e}-319$ & $1.5747 \mathrm{e}-217$ \\
& ACOC & 2.0000 & 2.0000 & 2.0000 \\
& iter & 8 & 9 & 8 \\
\hline$N 1$ & $\left|x_{k+1}-x_{k}\right|$ & $1.1580 \mathrm{e}-294$ & $2.9995 \mathrm{e}-186$ & $2.5139 \mathrm{e}-188$ \\
& $\left|f\left(x_{k+1}\right)\right|$ & $-1.3941 \mathrm{e}-1175$ & $1.5709 \mathrm{e}-741$ & $-1.9616 \mathrm{e}-752$ \\
& ACOC & 4.0000 & 3.9999 & 3.9999 \\
& iter & 5 & 5 & 5 \\
\hline \multirow{2}{*}{ N2 } & $\left|x_{k+1}-x_{k}\right|$ & $1.8263 \mathrm{e}-221$ & $2.2264 \mathrm{e}-141$ & $9.9149 \mathrm{e}-166$ \\
& $\left|f\left(x_{k+1}\right)\right|$ & $-7.2211 \mathrm{e}-1324$ & $3.833 \mathrm{e}-843$ & $4.8376 \mathrm{e}-993$ \\
& ACOC & 6.0000 & 5.9999 & 6.0000 \\
& iter & 4 & 4 & 4 \\
\hline \hline T0 & $\left|x_{k+1}-x_{k}\right|$ & $1.0499 \mathrm{e}-179$ & $5.7154 \mathrm{e}-125$ & $1.1477 \mathrm{e}-171$ \\
& $\left|f\left(x_{k+1}\right)\right|$ & $-1.0605 \mathrm{e}-535$ & $1.5612 \mathrm{e}-371$ & $2.2649 \mathrm{e}-514$ \\
& ACOC & 2.9999 & 2.9999 & 3.0000 \\
& iter & 6 & 6 & 6 \\
\hline T1 & $\left|x_{k+1}-x_{k}\right|$ & $5.8916 \mathrm{e}-102$ & $2.4805 \mathrm{e}-345$ & $3.3557 \mathrm{e}-461$ \\
& $\left|f\left(x_{k+1}\right)\right|$ & $-1.6331 \mathrm{e}-504$ & $1.2734 \mathrm{e}-1721$ & $-6.6081 \mathrm{e}-2305$ \\
& ACOC & 4.9999 & 4.9999 & 5.0000 \\
& iter & 4 & 5 & 5 \\
\hline T2 & $\left|x_{k+1}-x_{k}\right|$ & $7.0888 \mathrm{e}-282$ & $1.2632 \mathrm{e}-189$ & $3.4862 \mathrm{e}-261$ \\
& $\left|f\left(x_{k+1}\right)\right|$ & $-5.1956 \mathrm{e}-1966$ & $1.1288 \mathrm{e}-1320$ & $1.0076 \mathrm{e}-1826$ \\
& $\mathrm{ACOC}$ & 6.9999 & 6.9999 & 7.0000 \\
& iter & 4 & 4 & 4 \\
\hline \hline
\end{tabular}

Table 2: Numerical results from $f_{4}$ to $f_{6}$ for $N n$ and $T n$

This iterative method can be described by the following operator:

$$
M_{f}(x)=N_{f}(x)-\frac{f(x) f\left(N_{f}(x)\right)}{\left(f(x)-2 f\left(N_{f}(x)\right)\right) f^{\prime}(x)}
$$

where $N_{f}(x)=x-\frac{f(x)}{f^{\prime}(x)}$. Then, $x_{k+1}=M_{f}\left(x_{k}\right)$. The dynamics of the iterative method for the equation $f(x)=0$ depends on the fixed points of the operator $M_{f}$. Let us recall that a fixed point $p$ of an operator $M_{f}$ is said to be attractor if $\left|M_{f}^{\prime}(p)\right|<1$, and superattractor if $M_{f}^{\prime}(p)=0$.

It is easy to prove that the simple roots of $f$ are fixed points of $M_{f}$. The 
derivative of the operator is

$$
\begin{aligned}
M_{f}^{\prime}(x) & =-\frac{f\left(N_{f}(x)\right)}{f(x)-2 f\left(N_{f}(x)\right)}+\frac{f(x) f^{\prime \prime}(x)}{f^{\prime}(x)^{2}}+\frac{f(x) f\left(N_{f}(x)\right) f^{\prime \prime}(x)}{\left(f(x)-2 f\left(N_{f}(x)\right)\right) f^{\prime}(x)^{2}} \text { (13) } \\
& -\frac{f(x)^{2} f^{\prime}\left(N_{f}(x)\right) f^{\prime \prime}(x)}{\left(f(x)-2 f\left(N_{f}(x)\right)\right) f^{\prime}(x)^{3}}+\frac{f(x) f\left(N_{f}(x)\right)\left(f^{\prime}(x)-\frac{2 f(x) f^{\prime}\left(N_{f}(x)\right) f^{\prime \prime}(x)}{f^{\prime}(x)^{2}}\right)}{\left(f(x)-2 f\left(N_{f}(x)\right)\right)^{2} f^{\prime}(x)}
\end{aligned}
$$

By using the fact that if $x$ is near to a simple root of $f, f\left(N_{f}(x)\right)=O\left(f(x)^{2}\right)$, one obtains $M_{f}^{\prime}(x)=0$ in the roots of $f$, and then, these roots are superattracting points of the iteration operator. The dynamics of the method depends on the fixed points of $M_{f}$, which in general are not limited to the roots of $f$.

We will study the behavior of the method applied to real polynomials of degree 2 and 3 . The following result allows us to reduce the analysis to a few canonical examples.

Theorem 6 Let $f(x)$ be an analytic function, and let $T(x)=\alpha x+\beta$, with $\alpha \neq 0$, be an affine map. Let $g=f \circ T$. Then $M_{f}=T \circ M_{g} \circ T^{-1}$, that is, $M_{f}$ and $M_{g}$ are affine conjugated by $T$.

Proof: Let us show that $T^{-1}\left(M_{f}(x)\right)=M_{g}\left(T^{-1}(x)\right)$. If we denote $u=$ $T^{-1}(x)=\frac{x-\beta}{\alpha}$, then $g(u)=f(x)$. By using the chain rule, we have $g^{\prime}(u)=$ $f^{\prime}(T(u)) T^{\prime}(u)=\alpha f^{\prime}(x)$. Now, we prove that the Newton's iteration functions of $f$ and $g$ are affine conjugated by $T$ :

$$
N_{g}\left(T^{-1}(x)\right)=N_{g}(u)=u-\frac{g(u)}{g^{\prime}(u)}=\frac{x-\beta}{\alpha}-\frac{f(x)}{\alpha f^{\prime}(x)}=T^{-1}\left(N_{f}(x)\right)
$$

Thus, we have $g\left(N_{g}(u)\right)=g\left(T^{-1}\left(N_{f}(x)\right)\right)=f\left(N_{f}(x)\right)$, and finally,

$$
\begin{aligned}
M_{g}\left(T^{-1}(x)\right) & =M_{g}(u)=N_{g}(u)-\frac{g(u) g\left(N_{g}(u)\right)}{\left(g(u)-2 g\left(N_{g}(u)\right)\right) g^{\prime}(u)} \\
& =\frac{N_{f}(x)-\beta}{\alpha}-\frac{f(x) f\left(N_{f}(x)\right)}{\left(f(x)-2 f\left(N_{f}(x)\right)\right) \alpha f^{\prime}(x)} \\
& =T^{-1}\left(M_{f}(x)\right) .
\end{aligned}
$$

The theorem remains valid for $g(x)=c(f \circ T)(x)$, where $c \neq 0$.

This result allows us to reduce the study of the dynamics of $M_{f}$ on a given family of functions to simpler cases. Namely, if $f$ is a quadratic polynomial, the analysis can be reduced to one of the following particular cases: $f_{-}(x)=$ $x^{2}-1, f_{0}(x)=x^{2}$ or $f_{+}(x)=x^{2}+1$ depending on whether the polynomial has two, one (double) or no real roots. Similarly, any cubic polynomial reduces to one of the simplest cubic polynomials $f_{0}(x)=x^{3}, f_{+}(x)=x^{3}+x, f_{-}(x)=x^{3}-x$ or to a member of the one-parameter family of cubic maps $f_{\gamma}(x)=x^{3}+\gamma x+1$.

Let us now examine the different cases.

a. Quadratic polynomials 


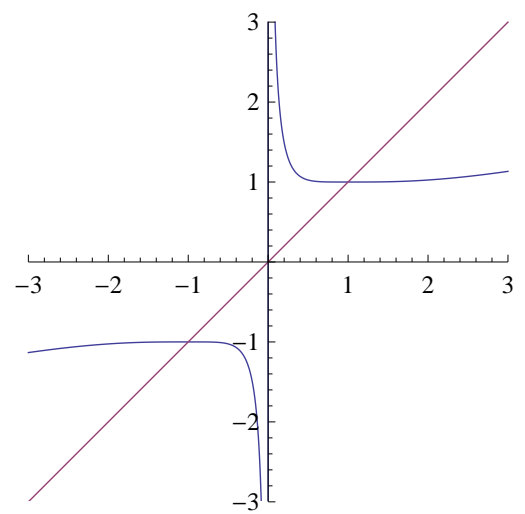

Figure 1: Fixed points of $M_{x^{2}-1}$

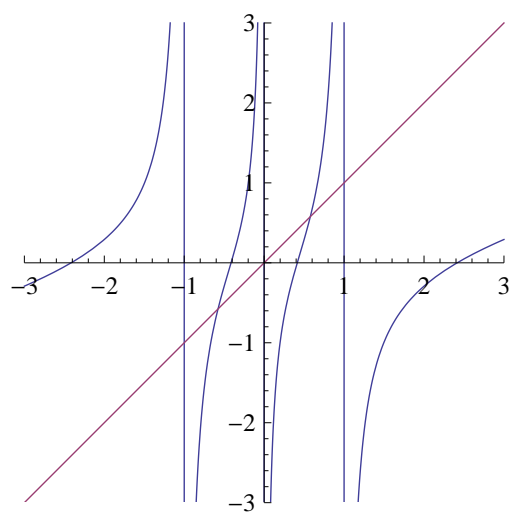

Figure 2: Fixed points of $M_{x^{2}+1}$

a1. Case $f_{0}(x)=x^{2}$

In this case $M_{f_{0}}(x)=\frac{t}{4}$ is a linear contraction. The double root of $f_{0}$ is the only fixed point of $f_{0}$. It is a global attractor, but not super-attractor.

a2. Case $f_{-}(x)=x^{2}-1$

The iteration function for $f_{-}$is $M_{f_{-}}(x)=\frac{x^{4}+6 x^{2}+1}{4 x\left(x^{2}+1\right)}$. Its only fixed points are the roots of $f_{-}$which are super-attracting. $M_{f_{-}}$has a vertical asymptote at $x=0$ (see Fig. 1).

a3. Case $f_{+}(x)=x^{2}+1$

The iteration function $M_{f_{+}}(x)=\frac{x^{4}-6 x^{2}+1}{4 x\left(x^{2}-1\right)}$ has two fixed points $x_{1,2}= \pm \frac{\sqrt{3}}{3}$, which are repelling because $M_{f_{+}}^{\prime}\left(x_{1,2}\right)=4>1$. There are 3 vertical asymptotes at $x=0, \pm 1$. The dynamics is chaotic because $f_{+}$has no real roots (see Fig. 2). 


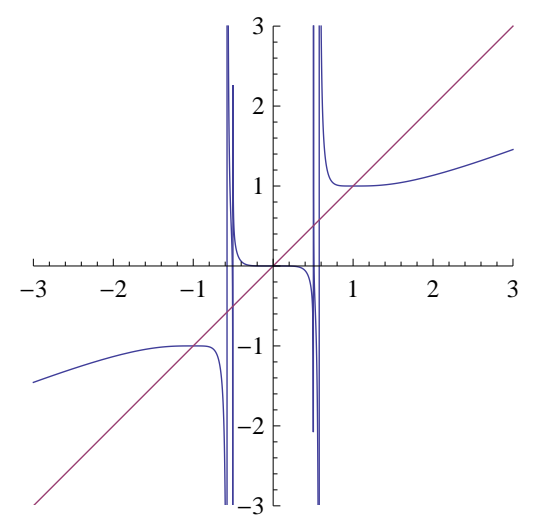

Figure 3: Fixed points and asymptotes of $M_{x^{3}-x}$

b. Cubic polynomials

b1. Case $f_{0}(x)=x^{3}$

In this case $M_{f_{0}}(x)=\frac{14 x}{33}$ is a linear contraction. The double root of $f_{0}$ is the only fixed point of $f_{0}$. It is a global attractor, but not super-attractor.

b2. Case $f_{+}(x)=x^{3}+x$

The iteration function in this case $M_{f_{+}}(x)=\frac{2 x^{5}\left(7 x^{4}-2 x^{2}-1\right)}{\left(3 x^{2}+1\right)\left(11 x^{6}+7 x^{4}+5 x^{2}+1\right)}$ has only the root of $f_{+}$as fixed super-attracting point. The dynamics is trivial.

b3. Case $f_{-}(x)=x^{3}-x$

In this case $M_{f_{-}}(x)=\frac{2 x^{5}\left(7 x^{4}+2 x^{2}-1\right)}{\left(3 x^{2}-1\right)\left(11 x^{6}-7 x^{4}+5 x^{2}-1\right)}$ has 5 fixed points, the 3 roots of $f_{-}: x_{1}=0, x_{2,3}= \pm 1$, that are super-attracting and another 2 extraneous fixed points, $x_{4,5} \simeq \pm 0.507592$, that are repelling. Moreover, $M_{f_{-}}$has asymptotes at the points $a_{1,2} \simeq \pm 0.504360$ and $a_{3,4} \simeq \pm 0.577350$.

b4. Case $f_{\gamma}(x)=x^{3}+\gamma x+1$

We are going to analyze the dynamics of $M_{f_{\gamma}}$ depending on the values of $\gamma$.

The function $f_{\gamma}$ has two critical points: $x_{\gamma+,-}= \pm \sqrt{-\frac{\gamma}{3}}$. The positive critical point is also a double root of $f_{\gamma}$ for the particular values of the parameter $\gamma_{*}=\frac{-3}{\sqrt{3} 4} \simeq-1.88988$. In principle, we consider three cases:

- If $\gamma<\gamma_{*}$, the polynomial $f_{\gamma}$ has three real roots.

- If $\gamma=\gamma_{*}$, the polynomial $f_{\gamma}$ has one positive double real root and a simple one which is negative.

- If $\gamma>\gamma_{*}$, the polynomial $f_{\gamma}$ has only one real root.

For $\gamma<\gamma_{*}$, the iterative method $M_{f_{\gamma}}$ has 5 fixed points separated by 4 vertical asymptotes (see Fig. 4). The fixed points are the 3 roots of $f_{\gamma}$, which are super-attracting and another 2 extraneous fixed points, which are repelling. 


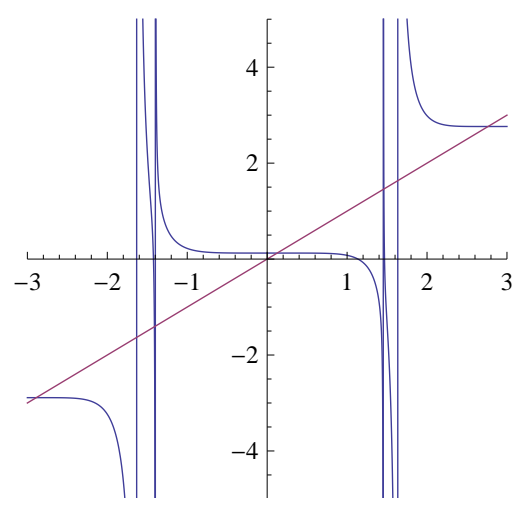

Figure 4: Fixed points and asymptotes of $M_{x^{3}-8 x+1}$

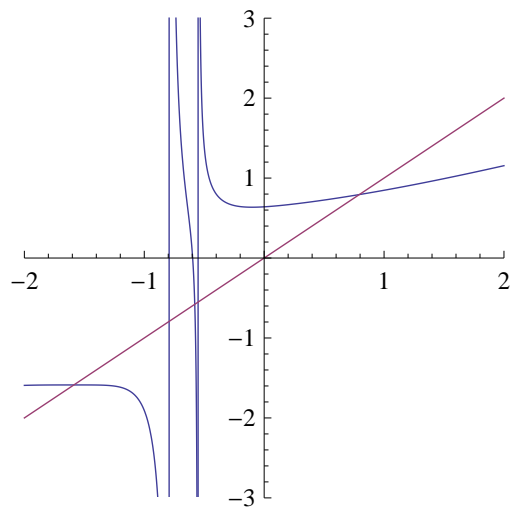

Figure 5: Fixed points and asymptotes of $M_{x^{3}-\gamma_{*} x+1}$

For $\gamma=\gamma_{*}$, the iterative method $M_{f_{\gamma}}$ has 3 fixed points separated by 2 vertical asymptotes (see Fig. 5). The fixed points are the simple root of $f_{\gamma}$, which is super-attracting, the double root, which is attracting and one extraneous fixed point, which is repelling.

For $\gamma_{*}<\gamma<-0.988$, the iterative method $M_{f_{\gamma}}$ has 5 fixed points (see Fig. 7 ). The only root of $f_{\gamma}$ is a super-attracting fixed point. The extraneous fixed points are repelling in general, but for the higher values of $\gamma$ in this interval, one of the fixed points becomes attracting until it joins another fixed point and both disappear. For this range of values, the method presents the typical behavior of the quadratic iteration producing bifurcations and period doubling in this region, failing to converge to the root of the function for some interval of starting points. Fig. 6 shows the values of the iterates after one hundred iterations depending on the $\gamma$ value. 


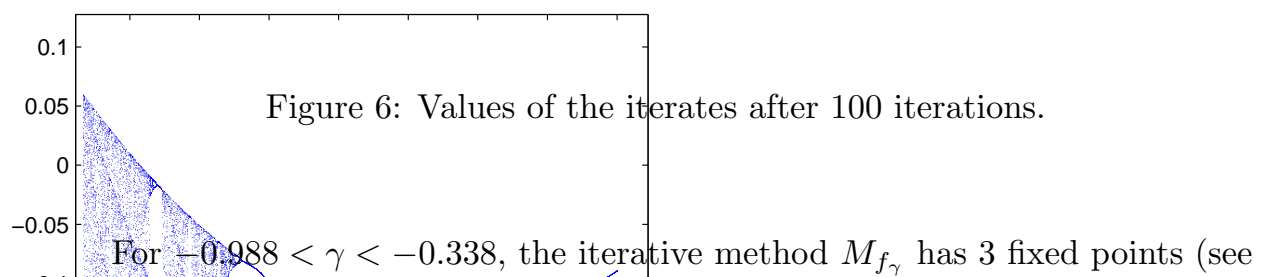

-0. Fig. 8). As before, the extraneous fixed points are repelling in general, but for -0.15 near -0.338 two fixed points join and disappear, producing quadratic chaos in a small interval of values of $\gamma$.

-0.2 For $2-0.388$, the only fixed point of the method is the root of the poly-0.25 ontal and it is super-attracting (see Fig. 9).

The iteration function has six asymptotes for $\gamma_{*}<\gamma<-1.225$, four asymptotes for $-1.225<\gamma<0$, two for $0<\gamma<0.655$, and none for $\gamma>0.655$. Then,

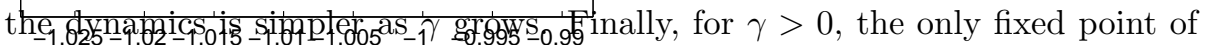
the method is again the root of the polynomial and it is super-attracting. There are no vertical asymptotes and the dynamics is trivial (see Fig. 10).

\section{Conclusions}

We have presented a technique that allows us to increase the order of an iterative method by performing additional steps that do not require the computation of new derivatives. The technique can be used repeatedly, increasing by two units the order of the method with each new step. By applying this technique, we have presented some new methods and compared them with known methods by using some test examples. The results confirm the convergence orders theoretically proved and show a good performance for these methods. The dynamical study shows that the studied method presents a good global behavior. 

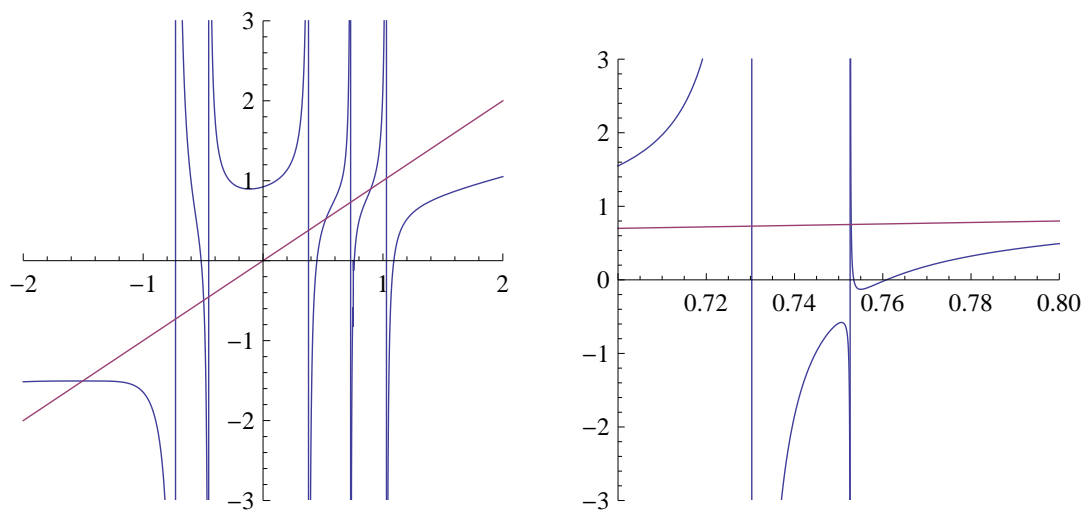

Figure 7: Fixed points and asymptotes of $M_{x^{3}-1.6 x+1}$. There are two very close asymptotes and a fixed point near the second asymptote as it can be seen in the image on the right.

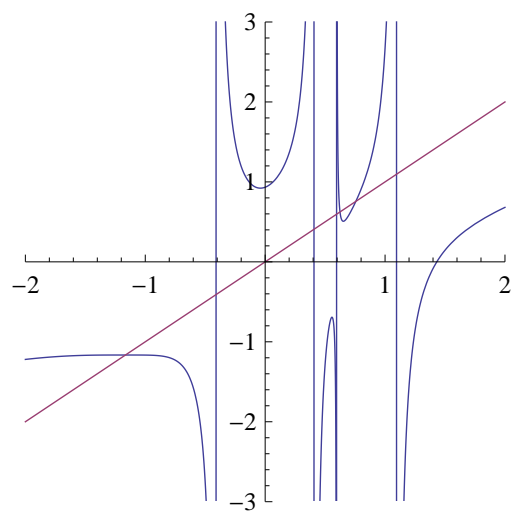

Figure 8: Fixed points and asymptotes of $M_{x^{3}-0.5 x+1}$. The function shape between the last two asymptotes suggests the presence of chaos. 


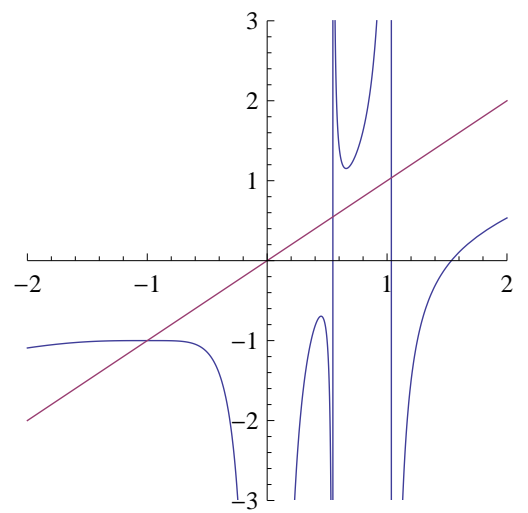

Figure 9: Fixed points and asymptotes of $M_{x^{3}+1}$

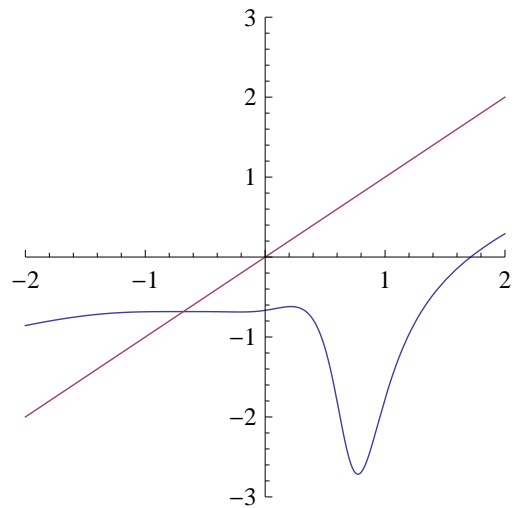

Figure 10: $M_{x^{3}+x+1}$ has a fixed point and no asymptotes 


\section{References}

[1] A. Cordero, J.L. Hueso, E. Martínez, J.R. Torregrosa, Increasing the convergence order of an iterative method for nonlinear systems, Applied Mathematics Letters 25, (2012), 2369-2374.

[2] J.F. Traub, Iterative methods for the solution of equations, Chelsea Publishing Company, New York, 1982.

[3] A.M. Ostrowski, Solutions of equations and systems of equations, Academic Press, New York-London, 1966.

[4] A. Cordero, E. Martínez, J.R. Torregrosa, Iterative methods of order four and five for systems of nonlinear equations, Journal of Computational and Applied Mathematics 231, (2009), 541-551.

[5] S. Amat, S. Busquier, A. Graub, M. Grau-Sánchez, Maximum efficiency for a family of Newton-like methods with frozen derivatives and some applications. Applied Mathematics and Computation, 219, (2013), 79547963.

[6] H.T. Kung, J.F. Traub, Optimal order of one-point and multi-point iteration. Applied Mathematics and Computation, 21, (1974), 643-651.

[7] S. Amat, S. Busquier, S. Plaza, Chaotic dynamics of a third-order Newtontype method, Journal of Mathematical Analysis and Applications 366-1 (2010) 2432

[8] Jarratt, P. Some fourth order multipoint iterative methods for solving equations. Math. Comput. 20, (1966), 434-437.

[9] M. Grau, J.L. Diaz-Barrero, An improvement to Ostrowski root-findfing method. Applied Mathematics and Computation 173(1), (2006), 450-456.

[10] Z. Hu, L. Guocai, L. Tian, An Iterative Method with Ninth-Order Convergence for Solving Nonlinear Equations. Int. J. Contemp. Math. Sciences, 6(1), (2011), 17-23.

[11] A. Cordero, J.R. Torregrosa, Variants of Newton's method using fifth-order quadrature formulas. Applied Mathematics and Computation 190, (2007), 686-698.

[12] S. Amat, S. Busquier, A. A.Magreñan Reducing Chaos and Bifurcations in Newton-Type Methods, Abstract and Applied Analysis, 2013, Article ID 726701, dx.doi.org/10.1155/2013/726701 